\title{
Large Format Heterodyne Arrays for Observing Far-Infrared Lines with SOFIA
}

\author{
${ }^{*}$ C. Walker ${ }^{1}$, C. Kulesa ${ }^{1}$, J. Kloosterman ${ }^{1}$, D. Lesser ${ }^{1}$, T. Cottam ${ }^{1}$, C. Groppi ${ }^{2}$, J. Zmuidzinas ${ }^{3}$, M. Edgar ${ }^{3}$, S. \\ Radford $^{3}$, P. Goldsmith ${ }^{4}$, W. Langer ${ }^{4}$, H. Yorke ${ }^{4}$, J. Kawamura ${ }^{4}$, I. Mehdi ${ }^{4}$, D. Hollenbach ${ }^{5}$, J. Stutzski ${ }^{6}$, \\ H. Huebers ${ }^{7}$, J.R. $\mathrm{Gao}^{8}$, C. Martin ${ }^{9}$ \\ ${ }^{1}$ Steward Observatory, University of Arizona, Tucson, AZ 85721 USA \\ ${ }^{2}$ School of Earth and Space Exploration, Arizona State University, Tempe, AZ 85287 USA \\ ${ }^{3}$ California Institute of Technology, Pasadena, CA 91125 USA \\ ${ }^{4}$ Jet Propulsion Laboratory, Pasadena, CA 91109 USA \\ ${ }^{5}$ SETI Institute, Mountain View, CA 94043 USA. \\ ${ }^{6}$ University of Cologne, Cologne, D-50937 Germany \\ ${ }^{7}$ DLR, Rutherfordstrasse 2, 12489 Berlin, Germany \\ ${ }^{8} \mathrm{TU}$ Delft, 2628 CJ Delft, Nederland \\ ${ }^{9}$ Oberlin College, Oberlin, OH 44074 USA.
}

\begin{abstract}
In the wavelength regime between 60 and 300 microns there are a number of atomic and molecular emission lines that are key diagnostic probes of the interstellar medium. These include transitions of [CII], [NII], [OI], $\mathrm{HD}, \mathrm{H}_{2} \mathrm{D}+, \mathrm{OH}, \mathrm{CO}$, and $\mathrm{H}_{2} \mathrm{O}$, some of which are among the brightest global and local far-infrared lines in the Galaxy. In Giant Molecular Clouds (GMCs), evolved star envelopes, and planetary nebulae, these emission lines can be extended over many arc minutes and possess complicated, often self absorbed, line profiles. High spectral resolution $\left(\mathrm{R}>10^{5}\right)$ observations of these lines at sub-arcminute angular resolution are crucial to understanding the complicated interplay between the interstellar medium and the stars that form from it. This feedback is central to all theories of galactic evolution. Large format heterodyne array receivers can provide the spectral resolution and spatial coverage to probe these lines over extended regions.

The advent of large format ( $\sim 100$ pixel) spectroscopic imaging cameras in the far-infrared (FIR) will fundamentally change the way astronomy is performed in this important wavelength regime. While the possibility of such instruments has been discussed for more than two decades, only recently have advances in mixer and local oscillator technology, device fabrication, micromachining, and digital signal processing made the construction of such instruments tractable. These technologies can be implemented to construct a sensitive, flexible, heterodyne array facility instrument for SOFIA. The instrument concept for StratoSTAR: Stratospheric Submm/THz Array Receiver includes a common user mounting, control system, IF processor, spectrometer, and cryogenic system. The cryogenic system will be designed to accept a frontend insert. The frontend insert and associated local oscillator system/relay optics would be provided by individual user groups and reflect their scientific interests. Rapid technology development in this field makes SOFIA the ideal platform to operate such a modular, continuously evolving instrument.
\end{abstract}

Keywords: TeraHertz, SOFIA, submillimeter, heterodyne, array receiver

\section{SCIENTIFIC MOTIVATION}

The THz portion of the spectrum is home to many spectral features that probe the life cycle of the interstellar medium (ISM). Table 1 identifies a subset of atomic and molecular species with transitions in the 2-6 $\mathrm{THz}$ frequency range. Three of the most important are [CII], [OI], and HD. All three are expected to extend over many arcminutes in the vicinity of star forming regions. Chemical and radiative transfer models can be used to predict how extensive the emission may be. Figure 1 illustrates the expected spectral appearance and extent of [OI] emission in the vicinity of a

Millimeter, Submillimeter, and Far-Infrared Detectors and Instrumentation for Astronomy V, edited by Wayne S. Holland, Jonas Zmuidzinas, Proc. of SPIE Vol. 7741, 77410Z

(c) 2010 SPIE · CCC code: 0277-786X/10/\$18 - doi: 10.1117/12.857811

Proc. of SPIE Vol. 7741 77410Z-1 
photo-dissociation (PDR) region, such as M17. Recent observations made with the HIFI instrument onboard Herschel (see Figure 2) indicates how complicated emission from PDRs can be, with many individual emission peaks observed along a single line of sight. Only heterodyne arrays can provide the high spectral resolution $\left(>10^{5}\right)$ needed to disentangle the emission from neutral and ionized gas over extended regions. THz receiver arrays on suborbital platforms like SOFIA are essential to providing the observational feedback necessary to constrain models and test our fundamental knowledge of the composition and physics of the ISM.

\section{Exciting Astronomy at 2-6 THz}

.... diverse, rich, and unexplored wavelength frontier...

\begin{tabular}{|cccc|}
\hline Species & Transition & THz & Importance \\
\hline \hline$[\mathrm{C} \mathrm{II}]$ & ${ }^{2} \mathrm{P}_{3 / 2 \rightarrow 1 / 2}$ & 1.9013 & Probe energetic PDR \\
{$[\mathrm{O} \mathrm{I}]$} & ${ }^{3} \mathrm{P}_{0 \rightarrow 1}$ & 2.06007 & surfaces of molecular clouds, \\
{$[\mathrm{N} \mathrm{II}]$} & ${ }^{3} \mathrm{P}_{2 \rightarrow 1}$ & 2.4622 & UC HII regions, AGB stars, \\
$\mathrm{H} \mathrm{I}$ & $\mathrm{n}=14 \rightarrow 13$ & 2.6811 & protoplanetary disks \\
{$[\mathrm{O}$ III $]$} & ${ }^{2} \mathrm{P}_{3 / 2 \rightarrow 1 / 2}$ & 3.3940 & \& molecular clouds. \\
{$[\mathrm{O} \mathrm{I}]$} & ${ }^{3} \mathrm{P}_{1 \rightarrow 2}$ & 4.74580 & \\
\hline \hline $\mathrm{CO}$ & $\mathrm{J}=17 \rightarrow 16$ & 1.9560 & Trace energetic molecular \\
& $\mathrm{J}=21 \rightarrow 20$ & 2.4139 & gas in star-forming regions, \\
& $\mathrm{J}=25 \rightarrow 24$ & 2.8703 & PDR's, SNR's, AGB ejecta. \\
\hline \hline $\mathrm{o}-\mathrm{H}_{2}^{18} \mathrm{O}$ & $2_{21} \rightarrow 1_{10}$ & 2.7416 & Probe enhanced $\mathrm{H}_{2} \mathrm{O}$ \\
$\mathrm{p}-\mathrm{H}_{2}^{18} \mathrm{O}$ & $2_{20} \rightarrow 1_{11}$ & 2.9389 & in shocks, outflows, disks. \\
\hline \hline $\mathrm{HD}$ & $0-0 \mathrm{R}(0)$ & 2.67499 & Cosmological nucleosynthesis, \\
& & & deuterium fractionation, \\
& & & thermal history of starforming \\
& & & regions, abundance of $\mathrm{H}_{2}$. \\
\hline \hline $\mathrm{OH}$ & $\Pi_{3 / 2 \rightarrow 1 / 2}$ & 1.83 & Ground state OH; general \\
& $\Pi_{7 / 2 \rightarrow 5 / 2}$ & 2.60 & $\mathrm{H}_{2} \mathrm{O}$ and oxygen chemistry. \\
\hline
\end{tabular}

Table 1: Subset of important atomic and molecular species found in the ISM at THz frequencies.

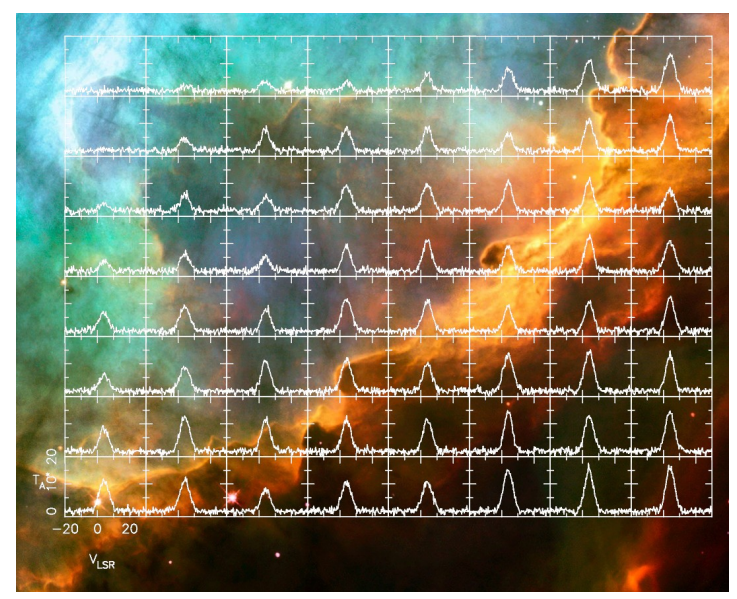

Figure 1: Model [OI] spectra in the vicinity of M17. The emission is bright and extended over many diffraction limited beams. 


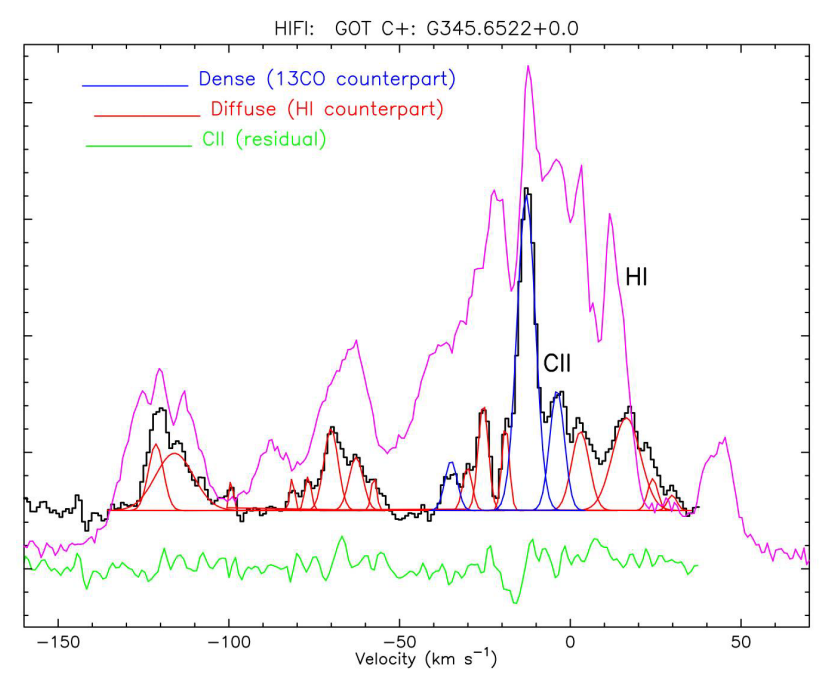

Figure 2. [CII] from Herschel HIFI's Galactic Observations of TeraHertz C+ Key Program [1] along with HI and CO.

\section{STRATOSTAR INSTRUMENT DESCRIPTION}

\subsection{Readiness Level of Heterodyne Array Technology}

SOFIA's optics can support a large number of THz array beams. Pictured in Figure 3 are the SOFIA beam footprints of 121 pixel $\mathrm{THz}$ heterodyne arrays near the frequencies of the [CII] and [OI] lines. Each circle represents a diffraction limited beam. The separation between adjacent pixels is $\sim 2$ full-width-half-maximums (FWHM).

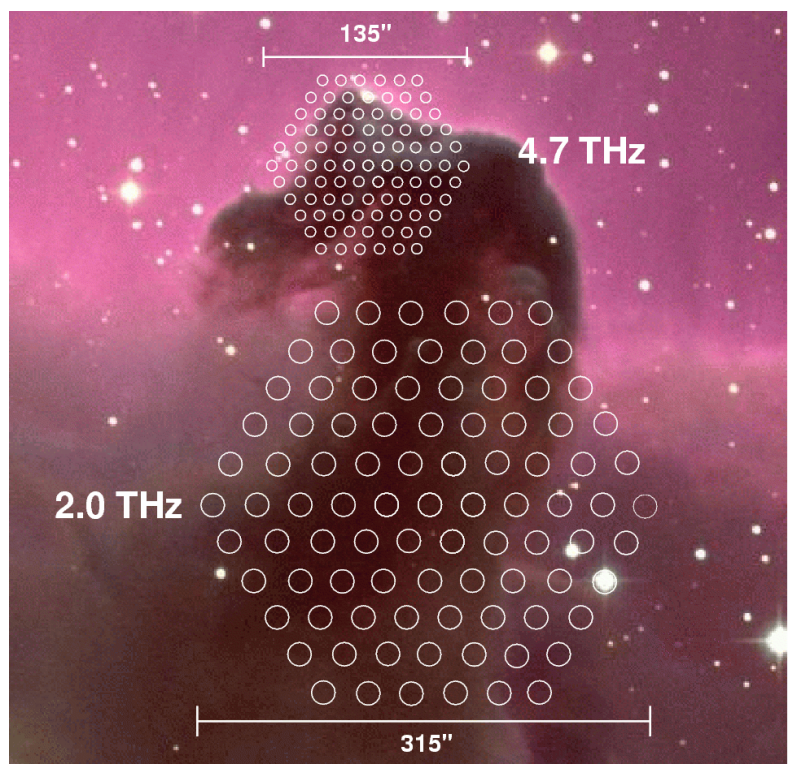

Figure 3: SOFIA THz Array Beams on the Sky.

$\mathrm{THz}$ arrays of this size are now possible due to a confluence of technological developments. Over the past $\sim 20$ years submillimeter-wave/ $\mathrm{THz}$ receivers have advanced from manually tuned, single pixel mixers with rack-size IF processors/spectrometers to multi-pixel, tunerless integrated systems with efficient, compact digital backend spectrometers (see Figure 4). Similarly, receiver sensitivity continues to improve, approaching $5 \mathrm{x}$ the quantum limit at $\sim 3 \mathrm{THz}$. 


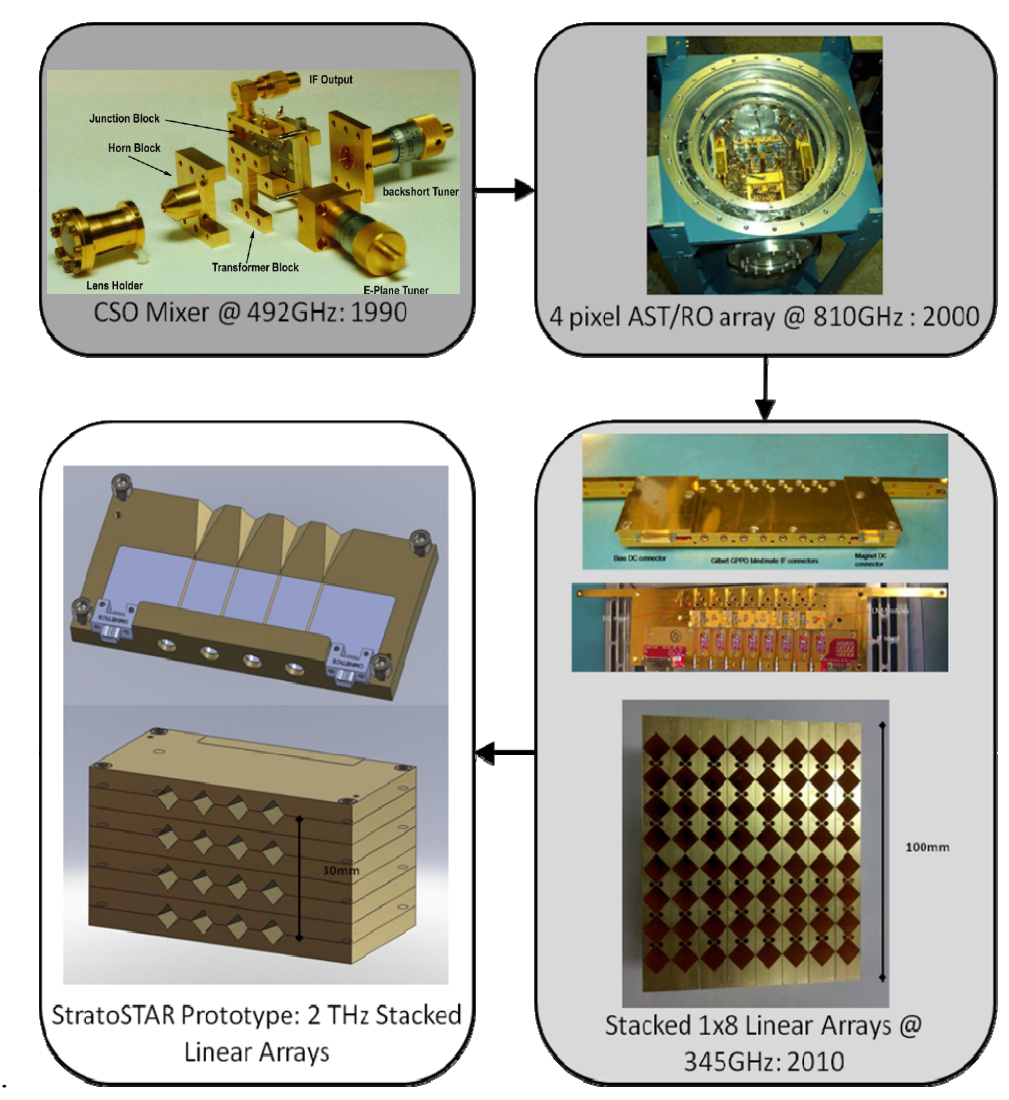

Figure 4. Above- Advances in SIS/HEB device fabrication, micromachining, and low-noise amplifiers has driven the evolution of waveguide receivers from individual, backshort tuned mixers to tunerless integrated arrays $[2,3,4]$. BelowLeft: GBT correlator spectrometer (ca. 1997) with 6.4GHz bandwidth. Right: SuperCam FFT/FPGA spectrometer (ca. 2007) with $16 \mathrm{GHz}$ of bandwidth.
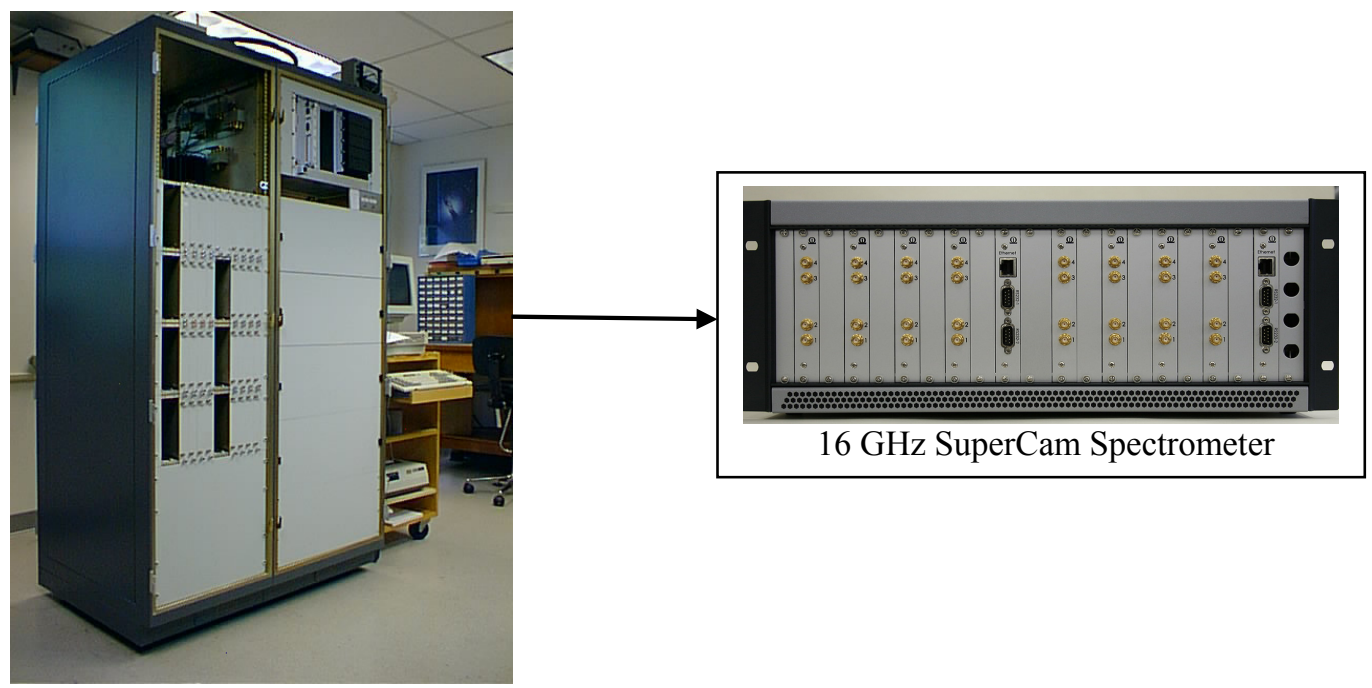

\subsection{GHz GBT Spectrometer [5]}

The development of the Herschel HIFI instrument has driven much of the development effort, particularly in the area of local oscillator (LO) power at high frequencies. Below is a plot (Figure 5) of existing and expected LO output power as a 
function of frequency. Below $\sim 2.7 \mathrm{THz}$ solid-state, frequency multipliers can provide sufficient output power to drive arrays. Above $2.7 \mathrm{THz}$, quantum cascade lasers (QCLs) can provide the necessary LO power to drive large arrays of THz mixers.

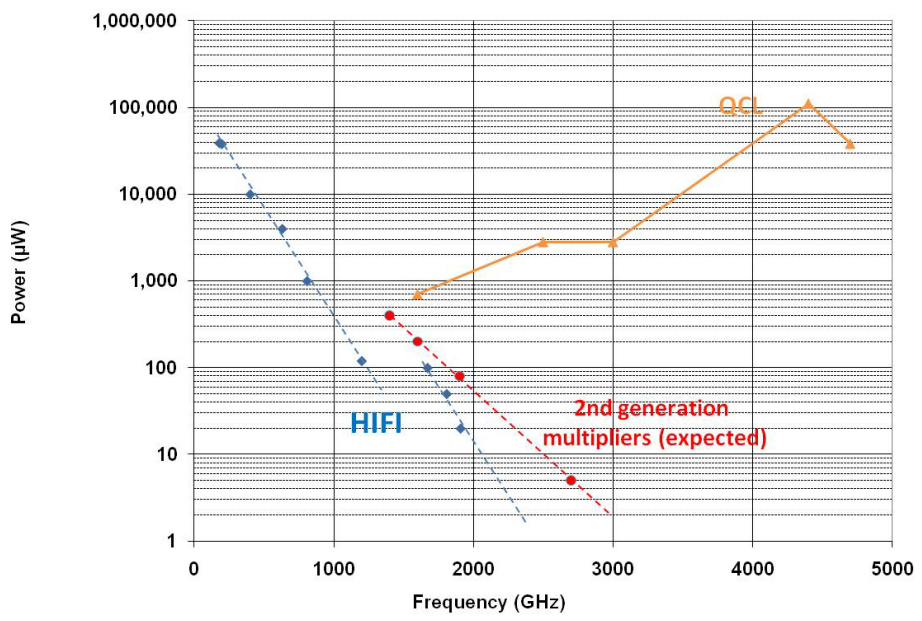

Figure 5. LO power vs. Frequency. HIFI utilized solid-state, frequency multiplied sources (blue curve). Quantum Cascade Lasers (red curve) can generate significant amounts of LO power at $\mathrm{THz}$ frequencies. It takes $\sim 1 \mu \mathrm{W}$ of $\mathrm{LO}$ power to drive one mixer.

\subsection{Instrument Concept}

A significant expenditure of cost and time in the development of SOFIA instrumentation goes into the FAA certification process. Where possible, second generation instruments should leverage from the $>10$ years of effort of first generation builders in this area. Fortunately, for the large format heterodyne arrays being considered here, much of the infrastructure (e.g. telescope mount, optics, control system) developed for the CASIMIR and GREAT single pixel instruments can be directly transferred or upgraded for use with arrays. The evolution to ever greater degrees of integration have resulted in array package sizes that are often $\leq$ what was used in the $1^{\text {st }}$ generation instruments. Indeed, recent design studies have shown that CASIMIR, as is, could host a pathfinder, 16 pixel StratoSTAR array. The array (see Figure 6) is composed of four, 1x4 subarrays of Hot Electron Bolometer (HEB) waveguide mixers optimized for the [CII] line at $1.9 \mathrm{THz}$. The mixer array can mount directly in a standard CASIMIR cryostat. The existing CASIMIR optics box can support the passage of all sixteen beams. The required mixer technology and all associated electronics (bias, IF amplifiers, IF processors, spectrometers) have already been developed either for CASIMIR, the Stratospheric THz Observatory (STO), or the 64 pixel- 345GHz SuperCam instrument for the Heinrich Hertz Telescope (HHT). A similar situation is also true for the GREAT instrument, which could also support an array receiver upgrade. For hosting much larger arrays ( $\sim 100$ pixels) a new cryostat would be needed, however this work would leverage heavily from the first generation instrumentation. 


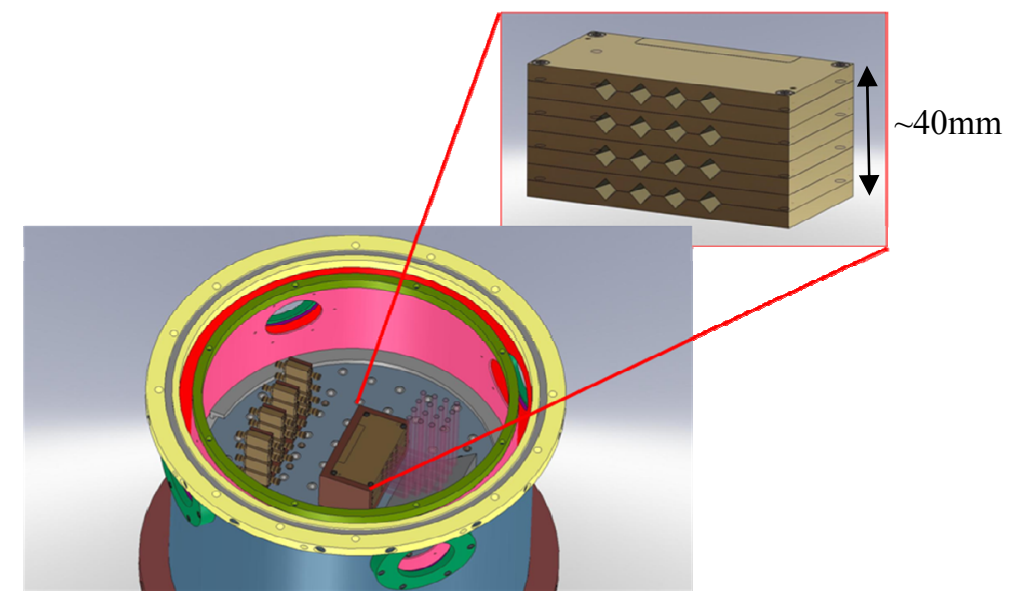

Figure 6. Sixteen pixel, $1.9 \mathrm{THz}$ Heterodyne Array integrated into a standard CASIMIR cryostat. The existing CASIMIR relay and calibration optics can support all 16 beams.

\section{CONCLUSION}

Large scale, high spectral resolution surveys are needed to help unravel the chemistry, physics, and evolution of the ISM. Knowledge of the ISM is essential to our understanding of star and planet formation in the Milky Way and beyond. Fortunately, technological evolution, driven in large part by Herschel, now makes it possible to construct large format, $\mathrm{THz}$ heterodyne arrays. When mounted to suborbital observing platforms, these $\mathrm{THz}$ arrays can be used for conducting the required surveys. Pathfinder arrays (10-20 pixels) can be realized on SOFIA by performing straightforward upgrades to CASIMIR and GREAT. Much larger arrays ( $\sim 100$ pixels) can be realized by leveraging experience gained from $1^{\text {st }}$ generation SOFIA instruments, STO, and ground-based arrays.

\section{REFERENCES}

[1] Langer et al. , "Galactic Observations of TeraHertz C+ Key Program”, ESLAB 2010 Conference (2010).

[2] Walker, C. K., Groppi, C., d'Aubigny, C., Kulesa, C., Hungerford, A., Jacobs, K., Graf, U., Schieder, R., \& Martin, C., "PoleSTAR: A 4-Pixel $810 \mathrm{GHz}$ Array Receiver for AST/RO", Proceedings of the 12th International Symposium on Space Tera- Hertz Technology, San Diego, CA, Eds. Mehdi \& McGrath, 540 (2001).

[3] Groppi, C. E. et al., "DesertSTAR: a 7 pixel $345 \mathrm{GHz}$ heterodyne array receiver for the Heinrich Hertz Telescope", SPIE, 4855, 330 (2003).

[4] Puetz, P., Hedden, A., Gensheimer, P., Golish, D., Groppi, C., Kulesa, K., Narayanan, G., Lichtenberger, A., Kooi, J., Wadefalk, N., Weinreb, S., Walker, C., "345 GHz Prototype SIS Mixer with Integrated MMIC LNA”, Int. J. Infrared Milli. Waves, 27, 1365 (2006).

[5] NRAO webpage," http://www.nrao.edu/engineering/spectrometer_gallery.shtml”" (2007). 